\title{
A Rigorous Replica Trick Approach to Anderson Localization in One Dimension
}

\author{
Abel Klein ${ }^{1, \star}$, Fabio Martinelli ${ }^{1, \star \star}$, and J. Fernando Perez ${ }^{2, \star \star \star}$ \\ 1 Department of Mathematics, University of California, Irvine, CA 92717, USA \\ 2 Instituto de Física, Universidade de São Paulo, Caixa Postal 20516, São Paulo, S.P., Brazil
}

\begin{abstract}
Let $H=-\Delta+V$ on $l^{2}(\mathbb{Z})$, where $V(x), x \in \mathbb{Z}$, are i.i.d.r.v.'s, and let $G_{L}(x, y ; E+i \eta)=\left\langle x\left|\left(H_{L}-(E+i \eta)\right)^{-1}\right| y\right\rangle$, where $H_{L}$ denotes the operator $H$ restricted to $\{-L,-L+1, \ldots, L\}$ with Dirichlet boundary conditions. We use a supersymmetric replica trick to prove that

$$
\mathbf{E}\left(\left|G_{L}(0, x ; E+i \eta)\right|^{2}\right) \leqq K \eta^{-2} \exp \left\{-m|\log \eta|^{-\sigma}|x|\right\}
$$

for some $m>0, \sigma>0, K<\infty$, uniformly in $L$ and $E$. This estimate, together with the usual necessary estimate on the density of states, implies zero conductivity and gives exponential localization by the Fröhlich, Martinelli, Scoppola, and Spencer method.
\end{abstract}

\section{Introduction}

The replica trick has been used in the physics literature to study the Anderson model by field theoretic methods [1]. It expresses the Green's function (or squared modulus of the Green's function) of the random Hamiltonian as a two-point function (or four-point function) of a field theory with $n$ independent replicas, which is then averaged over the randomness of the potential (introducing a coupling between the replicas). The quantities of interest are calculated, and then the limit is taken as the number of replicas $n \rightarrow 0$.

This $n \rightarrow 0$ limit is very mysterious. To circumvent it, Parisi and Sourlas [2] and McKane [3] introduced the supersymmetric replica trick, in which both Bose fields ("commuting variables") and spinless Fermi fields ("anti-commuting variables") are used in equal number of replicas, giving an effective total number of replicas $n=0$ without any need of taking a limit. This has been applied to the Anderson model [4].

\footnotetext{
* Research partially supported by NSF grants MCS83-01889 and INT 85-03418

$\star \star$ Permanent address: Dipartimento di Matematica, Universita di Roma "La Sapienza," Piazzale A. Moro, 2, I-00185 Rome, Italy. Partially supported by G.N.F.M. C.N.R.

$\star \star \star$ Partially supported by CNPq
} 
The supersymmetric replica trick is mathematically rigorous on a finite lattice. This was first explored by Klein and Perez [5] who used a supersymmetric cluster expansion to study the density of states in the Anderson model for large disorder or large energy. Subsequently Campanino and Klein [6] used the supersymmetric replica trick to obtain the sharpest results on the smoothness of the density of states in the one dimensional case. They studied the resulting one-dimensional supersymmetric field theory by means of a supersymmetric transfer matrix and did explicitly the integration over the anticommuting variables, obtaining a formula for the density of states from which they derived the smoothness properties.

In this article we obtain an estimate that gives exponential decay for the averaged squared modulus of the Green's function in one dimension. We use the supersymmetric replica trick to rewrite it as a four-point function of a one dimensional supersymmetric field theory and we do explicitly the integration over the anticommuting variables. We then extract the exponential decay from the resulting formula.

This estimate, combined with the usual necessary estimate on the density of states, gives zero conductivity and exponential localization. The former is almost immediate and the latter is obtained by the Fröhlich, Martinelli, Scoppola, and Spencer [7] method, where our estimate replaces Fustenberg's theorem. For previous results see the reviews $[13,14]$ and references in $[10]$.

It is our hope and part of our motivation in this work to prove a similar estimate in higher dimensions, at least for high disorder or low energy.

The one-dimensional Anderson model is given by the random Hamiltonian $H=H_{0}+V$ on $l^{2}(\mathbb{Z})$, where

$$
\left(H_{0} u\right)(x)=\frac{1}{2}(u(x+1)+u(x-1))
$$

and $V(x), x \in \mathbb{Z}$, are independent identically distributed random variables with common probability distribution $v$. If $\Lambda_{L}=\{-L,-L+1, \ldots, L\}, H_{L}$ will denote the operator $H$ restricted to $l^{2}\left(\Lambda_{L}\right)$ with boundary condition $u(x)=0$ for $x$ not in $\Lambda_{L}$. For $z \in \mathbb{C}, \operatorname{Im} z \neq 0$, let

$$
\begin{aligned}
& G_{L}(x, y ; z)=\left\langle x\left|\left(H_{L}-z\right)^{-1}\right| y\right\rangle \text { for } x, y \in \Lambda_{L}, \\
& G(x, y ; z)=\left\langle x\left|(H-z)^{-1}\right| y\right\rangle \quad \text { for } \quad x, y \in \mathbb{Z} ;
\end{aligned}
$$

notice that $G_{L}(x, y ; z) \rightarrow G(x, y ; z)$ as $L \rightarrow \infty$.

If $\mu$ is a probability measure, its characteristic function is given by $\hat{\mu}(t)$ $=\int e^{-i t v} d \mu(v)$.

We are now ready to state our main result.

Main Theorem. Let $v=\alpha v_{1}+(1-\alpha) v_{2}$, where $0 \leqq \alpha<1, v_{1}$ is an arbitrary probability measure, and $v_{2}$ satisfies

and

$$
\int_{0}^{\infty}\left|\hat{v}_{2}(t)\right|^{p_{1}}|\log t| d t<\infty \quad \text { for some } \quad p_{1}<\infty
$$

$$
\int_{0}^{\infty}\left|\hat{v}_{2}(t)\right|^{p} d t \leqq C p^{-(1 / \sigma)} \quad \text { for some } \quad \sigma>0, \quad C<\infty
$$


and all $p_{2} \leqq p<\infty$ for some $p_{2}$. Then there exists $m>0$ and $\eta_{0}>0$ such that

$$
\mathbf{E}\left(\left|G_{L}(0, x ; E+i \eta)\right|^{2}\right) \leqq K \eta^{-2} \exp \left\{-m|\log \eta|^{-\sigma}|x|\right\}
$$

for all $L, x \in \Lambda_{L}, E, \eta \in \mathbb{R}$ with $0<\eta \leqq \eta_{0}$ and some $K<\infty$.

Notice that (1.2) is implied by (1.1) plus

$\left|\hat{v}_{2}(t)\right| \leqq 1-c|t|^{\sigma}$ in a neighborhood of zero for some $\sigma>0$ and $c>0$,

and that there are singular continuous $v_{2}$ satisfying these conditions [8].

The other ingredient for localization is an estimate on the density of states. In a form suitable for our needs it can be stated as follows:

For every $\alpha, \beta>0$ and $E_{0}>0$, there exists $\delta>0$ and $L_{0}>0$ such that for all $|E| \leqq E_{0}$ and $L \geqq L_{0}$, we have

$$
\mathbf{P}\left\{\operatorname{dist}\left(E, \sigma\left(H_{L}\right)\right)<e^{-\alpha L^{\beta}}\right\} \leqq e^{-\delta L^{\beta}} .
$$

If $v$ has a bounded density, this is a simple consequence of a result of Wegner [9]. It can be proven with the use of the supersymmetric replica trick by the methods of Campanino and Klein [6] if either $\int_{0}^{\infty}|\hat{v}(t)|^{p} t^{\kappa^{-1 / 2}} d t<\infty$ for some $p<\infty$ and $\kappa>0$, or if $v=\alpha v_{1}+(1-\alpha) v_{2}, \int|v| d v_{1}<\infty$ and $(1+|t|)^{\varepsilon} \hat{v}_{2}^{(j)}(t)$ bounded for some $\varepsilon>0$ and $j=0,1$. Using different methods, Carmona, Klein, and Martinelli [10] proved (1.4) for any $v$ such that $\int|v|^{\varepsilon} d v<\infty$ for some $\varepsilon>0$ and its support contains more than one point. As a consequence they were able to prove exponential localization for any such $v$, in particular if $v$ is a Bernoulli measure.

Corollary 1. Suppose (1.3) and (1.4) hold. Then given $E_{0}>0$ there exists $\varepsilon>0$, $C_{1}<\infty, m_{1}<\infty, \eta_{1}>0$ such that

$$
\mathbf{E}\left(|G(0, x ; E+i \eta)|^{2}\right) \leqq C_{1} \eta^{-(2-\varepsilon)} \exp \left\{-m_{1}|\log \eta|^{-\sigma}|x|\right\}
$$

for all $x \in \mathbb{Z},|E| \leqq E_{0}$ and $0<\eta \leqq \eta_{1}$. In particular the conductivity

for all $E \in \mathbb{R}$.

$$
\sigma(E)=\lim _{\eta \downarrow 0} \eta^{2} \sum_{x=-\infty}^{\infty}|x|^{2} \mathbf{E}\left(|G(0, x ; E+i \eta)|^{2}\right)=0
$$

Corollary 2. Suppose (1.3) and (1.4) hold. Then given $E_{0}>0$ there exist $d_{1}, d_{2}>0$ and $L_{1}>0$ such that

$$
\mathbf{P}\left\{\left|G_{L}(0, L ; E)\right|+\left|G_{L}(0,-L ; E)\right|>\exp \left(-d_{1} L^{(\sigma+1)^{-1}}\right)\right\} \leqq \exp \left(-d_{2} L^{(\sigma+1)^{-1}}\right)
$$

for all $|E| \leqq E_{0}$ and $L \geqq L_{1}$.

Carmona, Klein, and Martinelli [10] have obtained a sharper estimate in great generality by different methods.

Inequalities (1.5) and (1.4) are all that is needed to apply the Fröhlich, Martinelli, Scoppola, and Spencer method [7] in one dimension, so we get

Corollary 3. Suppose (1.3) and (1.4) hold. Then the spectrum of $H$ is pure point with probability one and the corresponding eigenfunctions decay exponentially. 
This article is organized as follows: in the next section we introduce the supersymmetric replica trick and perform the integration over the anticommuting variables. The Main Theorem is proven in Sect. 3. Corollary 2 is proven in Sect. 4 and Corollary 3 in Sect. 3.

Note. Kotani [15] has proved that our estimate (1.3) implies $\gamma(E)>0$ for a.e. $E$, where $\gamma(E)$ is the Lyapunov exponent. He also showed that (1.3) and (1.4) combined imply $\gamma(E)>0$ everywhere. Of course, this also follows from Corollary 3.

\section{The Supersymmetric Replica Trick}

The supersymmetric replica trick [2-6] says that, if $x_{1}, x_{2} \in \Lambda_{L}, z \in \mathbb{C}$ with $\operatorname{Im} z>0$,

$$
G_{L}\left(x_{1}, x_{2} ; z\right)=i \int \psi\left(x_{1}\right) \bar{\psi}\left(x_{2}\right) \exp \left\{-i \sum_{y=-L}^{L} \Phi(y) \cdot\left[\left(H_{L}-z\right) \Phi\right](y)\right\} D_{L} \Phi,
$$

where $\Phi(x)=(\phi(x), \psi(x), \bar{\psi}(x))$ is a "superfield" with $\phi(x) \in \mathbb{R}^{2}$ and $\psi(x), \bar{\psi}(x)$ anticommuting "variables" [i.e., elements of a Grassmann algebra: $\psi(x)^{2}=\bar{\psi}(x)^{2}$ $=\bar{\psi}(x) \psi(x)+\psi(x) \bar{\psi}(x)=0], \quad \Phi(x) \cdot \Phi(y)=\phi(x) \cdot \phi(y)+\frac{1}{2}(\bar{\psi}(x) \psi(y)+\bar{\psi}(y) \psi(x))$, and

$$
D_{L} \Phi=\prod_{y=-L}^{L} d \Phi(y) \quad \text { with } \quad d \Phi(y)=\frac{1}{\pi} d \bar{\psi}(y) d \psi(y) d^{2} \phi(y)
$$

(see $[11,12])$. Notice that $\int e^{-\Phi(y) \cdot \Phi(y)} d \Phi(y)=1$.

Since we are working in a finite lattice, (2.1) is mathematically rigorous. To compute functions of $\psi, \bar{\psi}$ we expand in power series that terminate after a finite number of terms due to the anticommutativity. All $\{\psi(y), \bar{\psi}(y) ; y=-L,-L+1, \ldots, L\}$ anticommute. The linear functional denoted by $\int \cdot d \tilde{\psi}(y) d \psi(y)$ (it is not an actual integral) is defined by [11]. To simplify our equations, we will abuse the notation by writing $\Phi(y)^{2} \equiv \Phi(y) \cdot \Phi(y), \phi(y)^{2}$ $\equiv \phi(y) \cdot \phi(y)$.

Since $\bar{G}_{L}\left(x_{1}, x_{2} ; z\right)=G_{L}\left(x_{1}, x_{2} ; \bar{z}\right)$, and since for $\operatorname{Im} \bar{z}<0$ we have, similarly to $(2.1)$,

$$
G_{L}\left(x_{1}, x_{2} ; \bar{z}\right)=-i \int \psi\left(x_{1}\right) \bar{\psi}\left(x_{2}\right) \exp \left\{i \sum_{y=-L}^{L} \Phi(y) \cdot\left[\left(H_{L}-\bar{z}\right) \Phi\right](y)\right\} D_{L} \Phi,
$$

we get [4], for any $E \in \mathbb{R}$ and $\eta>0$,

$$
\begin{aligned}
\left|G_{L}\left(x_{1}, x_{2} ; E+i \eta\right)\right|^{2}= & -\int \psi_{+}\left(x_{1}\right) \psi_{-}\left(x_{1}\right) \bar{\psi}_{+}\left(x_{2}\right) \bar{\psi}_{-}\left(x_{2}\right) \\
& \times \exp \left\{-i \sum_{y=-L}^{L} V(y)\left[\Phi_{+}(y)^{2}-\Phi_{-}(y)^{2}\right]\right. \\
& +i E \sum_{y=-L}^{L}\left[\Phi_{+}(y)^{2}-\Phi_{-}(y)^{2}\right] \\
& -\eta \sum_{y=-L}^{L}\left[\Phi_{+}(y)^{2}+\Phi_{-}(y)^{2}\right] \\
& \left.-i \sum_{y=-L}^{L-1}\left[\Phi_{+}(y) \cdot \Phi_{+}(y+1)-\Phi_{-}(y) \cdot \Phi_{-}(y+1)\right]\right\} \\
& \times D_{L} \Phi_{+} D_{L} \Phi_{-},
\end{aligned}
$$

where $\Phi_{+}, \Phi_{-}$are two replicas of the superfield $\Phi$. 
If we now assume that $\int v^{2} d v<\infty$, so $\hat{v}$ is twice differentiable with bounded first and second derivatives, we can average over the random potential in (2.2) to obtain:

Lemma 2.1. Let $\int v^{2} d v<\infty, E \in \mathbb{R}, \eta>0$. Then for all $L$ and $x_{1}, x_{2} \in \Lambda_{L}$, we have

$$
\begin{aligned}
\mathbf{E}\left(\left|G_{L}\left(x_{1}, x_{2} ; E+i \eta\right)\right|^{2}=\right. & -\int \psi_{+}\left(x_{1}\right) \psi_{-}\left(x_{1}\right) \bar{\psi}_{+}\left(x_{2}\right) \bar{\psi}_{-}\left(x_{2}\right) \\
& \times \prod_{y=-L}^{L} \Gamma\left(\Phi_{+}(y)^{2}, \Phi_{-}(y)^{2} ; E, \eta\right) \\
& \times \exp \left\{-i \sum_{y=-L}^{L-1}\left[\Phi_{+}(y) \cdot \Phi_{+}(y+1)\right.\right. \\
& \left.\left.-\Phi_{-}(y) \cdot \Phi_{-}(y+1)\right]\right\} D_{L} \Phi_{+} D_{L} \Phi_{-},
\end{aligned}
$$

where $\Gamma(r, s ; E, \eta)=\hat{v}(r-s) e^{i E(r-s)-\eta(r+s)}$.

We will now use the fact that the right-hand side of (2.3) is a 4-point function of a one-dimensional superfield theory to do explicitly the integration over the anticommuting variables; for simplicity we will take $x_{1}=0, x_{2}=x$ with $0 \leqq x \in \Lambda_{L}$.

Consider (we will suppress $E, \eta$ in the formulas)

$$
\begin{aligned}
\Delta_{n}\left(\Phi_{+}, \Phi_{-}\right)= & \exp \left\{-(\eta / 2)\left[\Phi_{+}^{2}+\Phi_{-}^{2}\right]\right\} \int \exp \left\{-i\left[\Phi_{+} \cdot \Phi_{+}(1)-\Phi_{-} \cdot \Phi_{-}(1)\right.\right. \\
& \left.+\sum_{j=1}^{n-1}\left(\Phi_{+}(j) \cdot \Phi_{+}(j+1)-\phi_{-}(j) \cdot \Phi_{-}(j+1)\right]\right\} \\
& \times \prod_{j=1}^{n} \Gamma\left(\Phi_{+}(j)^{2}, \Phi_{-}(j)^{2}\right) \prod_{j=1}^{n} d \Phi_{+}(j) d \Phi_{-}(j) .
\end{aligned}
$$

It is easy to see that $\Delta_{n}\left(\Phi_{+}, \Phi_{-}\right)$is separately supersymmetric in $\Phi_{+}$and $\Phi_{-}$, hence there exists a function $\Omega_{n}(r, s)$ of class $C^{2}$ on $[0, \infty) \times[0, \infty)$ (see [12, Sect. 4]) such that $\Delta_{n}\left(\Phi_{+}, \Phi_{-}\right)=\Omega_{n}\left(\Phi_{+}^{2}, \Phi_{-}^{2}\right)$.

Thus (2.3) can be rewritten as

$$
\begin{aligned}
\mathbf{E}\left(\left|G_{L}(0, x ; E+i \eta)\right|^{2}\right)= & -\int \Omega_{L}\left(\Phi_{+}(0)^{2}, \Phi_{-}(0)^{2}\right) \\
& \times \psi_{+}(0) \psi_{-}(0) \exp \left\{(\eta / 2)\left[\Phi_{+}(0)^{2}+\Phi_{-}(0)^{2}\right]\right\} \\
& \times \prod_{y=0}^{x} \Gamma\left(\Phi_{+}(y)^{2}, \Phi_{-}(y)^{2}\right) \\
& \times \exp \left\{-i \sum_{y=0}^{x-1}\left[\Phi_{+}(y) \cdot \Phi_{+}(y+1)-\Phi_{-}(y) \cdot \Phi_{-}(y+1)\right]\right\} \\
& \times \exp \left\{(\eta / 2)\left[\Phi_{+}(x)^{2}+\Phi_{-}(x)^{2}\right]\right\} \bar{\psi}_{+}(x) \bar{\psi}_{-}(x) \\
& \times \Omega_{L-x}\left(\Phi_{+}(x)^{2}, \Phi_{-}(x)^{2}\right) \prod_{y=0}^{x} d \Phi_{+}(y) d \Phi_{-}(y) .
\end{aligned}
$$


We can now do explicitly the integration over the anticommuting variables in (2.4) to get

$$
\begin{aligned}
\mathbf{E}\left(\left|G_{L}(0, x ; E+i \eta)\right|^{2}=\right. & \frac{1}{\pi^{2}}\left(\frac{-1}{2 \pi}\right)^{2 x} \int \Omega_{L}\left(\phi_{+}(0)^{2}, \phi_{-}(0)^{2}\right) \\
& \times \exp \left\{(\eta / 2)\left[\phi_{+}(0)^{2}+\phi_{-}(0)^{2}\right]\right\} \prod_{y=0}^{x} \Gamma\left(\phi_{+}(y)^{2}, \phi_{-}(y)^{2}\right) \\
& \times \exp \left\{-i \sum_{y=0}^{x-1}\left[\phi_{+}(y) \cdot \phi_{+}(y+1)-\phi_{-}(y) \cdot \phi_{-}(y+1)\right]\right\} \\
& \times \exp \left\{(\eta / 2)\left[\phi_{+}(x)^{2}+\phi_{-}(x)^{2}\right]\right\} \Omega_{L-x}\left(\phi_{+}(x)^{2}, \phi_{-}(x)^{2}\right) \\
& \times \prod_{y=0}^{x} d^{2} \phi_{+}(y) d^{2} \phi_{-}(y) .
\end{aligned}
$$

We will now change to polar coordinates and recall that

$$
J_{0}(s)=J_{0}(-s)=\frac{1}{2 \pi} \int_{0}^{2 \pi} e^{-i s \cos \theta} d \theta
$$

is the Bessel function of order zero. We will use $\Gamma(E, \eta)$ to also denote the operator multiplication by the function $\Gamma(\cdot, \cdot ; E, \eta)$, and $K(\eta)$ to denote multiplication by the function $f(r, s)=e^{(\eta / 2)(r+s)}$.

We thus have

Lemma 2.2. Let $\int v^{2} d v<\infty, E \in \mathbb{R}, \eta>0$. Then for all $L$ and $0 \leqq x \in \Lambda_{L}$, we have

$$
\mathbf{E}\left(\left|G_{L}(0, x ; E+i \eta)\right|^{2}\right)=\left\langle\bar{\Omega}_{L}, K(\eta) \Gamma(E, \eta)(S \Gamma(E, \eta))^{x} K(\eta) \Omega_{L-x}\right\rangle,
$$

where $\langle$,$\rangle is the inner product in the complex Hilbert space$

$$
\mathscr{K}=\left\{f:[0, \infty)^{2} \rightarrow C \text { measurable; }\|f\|^{2}=4 \int_{0}^{\infty} \int_{0}^{\infty}\left|f\left(r_{+}^{2}, r_{-}^{2}\right)\right|^{2} r_{+} r_{-} d r_{+} d r_{-}<\infty\right\}
$$

and $S$ the operator on $\mathscr{K}$ given by

$$
(S f)\left(r_{+}^{2}, r_{-}^{2}\right)=-\int_{0}^{\infty} \int_{0}^{\infty} J_{0}\left(r_{+} s_{+}\right) J_{0}\left(r_{-} s_{-}\right) f\left(s_{+}^{2}, s_{-}^{2}\right) s_{+} s_{-} d s_{+} d s_{-} .
$$

\section{Proof of the Main Theorem}

Let us first assume that $\int v^{2} d v<\infty$. From (2.5) we get

$$
\begin{aligned}
\mathbf{E}\left(\left|G_{L}(0, x ; E+i \eta)\right|^{2}\right) \leqq & \| K(\eta) \Gamma(E, \eta)\left(S \Gamma(E, \eta)^{x} K(\eta) \|\right. \\
& \times\left\|\Omega_{L}(E, \eta)\right\|\left\|\Omega_{L-x}(E, \eta)\right\|,
\end{aligned}
$$

where all the norms are in $\mathscr{K}$, the first one being the operator norm on $\mathscr{K}$.

We first prove

Lemma 3.1. For all $n$ and all $E \in \mathbb{R}, \eta>0$,

$$
\left\|\Omega_{n}(E, \eta)\right\| \leqq \eta^{-1}
$$


Proof of Lemma 3.1. We will undo (2.5). If we go through its derivation we can check that

$$
\left\|\Omega_{n}(E, \eta)\right\|^{2}=\left\langle\Omega_{n}(E, \eta), \Omega_{n}(E, \eta)\right\rangle=\mathbf{E}\left(\left\langle 0\left|\left(H_{0, n}+W-i \eta\right)^{-1}\right| 0\right\rangle\right),
$$

where $W(j), j=-n,-n+1, \ldots, n$ are independent random variables such that $W(0) \equiv 0$ and both $W(j)+E$ and $-W(-j)-E$ are distributed like $v$ for $j=1,2, \ldots, n$, and $H_{0, n}$ is the operator $H_{0}$ restricted to $\Lambda_{n}$ with zero boundary conditions.

Thus $\left\|\Omega_{n}(E, \eta)\right\| \leqq \eta^{-1}$.

We must now estimate $\left\|K(\eta) \Gamma(E, \eta)(S \Gamma(E, \eta))^{x} K(\eta)\right\|$. Since $K(\eta) \Gamma(E, \eta)$ is multiplication by a function $\leqq 1$ in modulus, and since $S$ is unitary on $\mathscr{K}$ (see the discussion in the proof of the following lemma), it suffices to prove

Lemma 3.2. Let $v$ be as in the Main Theorem. Then there exists $b>0$ and $\eta_{0}>0$ such that

$$
\|\Gamma(E, \eta) S \Gamma(E, \eta)\| \leqq \exp \left\{-b|\log \eta|^{-\sigma}\right\}
$$

for all $E \in \mathbb{R}$ and $0<\eta \leqq \eta_{0}$.

Proof. Let us define the double Hankel transform of order zero by

$$
\left(H_{0} g\right)\left(r_{+}, r_{-}\right)=\int_{0}^{\infty} \int_{0}^{\infty} \sqrt{r_{+} s_{+}} \sqrt{r_{-} s_{-}} J_{0}\left(r_{+} s_{+}\right) J_{0}\left(r_{-} s_{-}\right) g\left(s_{+}, s_{-}\right) d s_{+} d s_{-} .
$$

Then $H_{0}$ is unitary on $L^{2}\left([0, \infty)^{2}, d r_{+} d r_{-}\right)$and $\left\|H_{0} g\right\|_{\infty} \leqq\|g\|_{1}$, so by the Riesz Convexity Theorem we get a Hausdorff-Young inequality for $H_{0}$ :

$$
\left\|H_{0} g\right\|_{p^{\prime}} \leqq\|g\|_{p} \quad \text { for } \quad 1 \leqq p \leqq 2, \quad \frac{1}{p}+\frac{1}{p^{\prime}}=1
$$

(see the discussion in [6] for a similar situation). Here all $L^{P}$ norms are with respect to $\left([0, \infty)^{2}, d r_{+} d r_{-}\right)$.

From (2.6) we have that

$$
\sqrt{r_{+} r_{-}}(S f)\left(r_{+}^{2}, r_{-}^{2}\right)=-H_{0}\left(\sqrt{s_{+} s_{-}} f\left(s_{+}^{2}, s_{-}^{2}\right)\right)\left(r_{+}, r_{-}\right) .
$$

From this it follows that $S$ is unitary on $\mathscr{K}$ and we have a Hausdorff-Young inequality for $S$ :

$$
\left\|\sqrt{r_{+} r_{-}}(S f)\left(r_{+}^{2}, r_{-}^{2}\right)\right\|_{p^{\prime}} \leqq\left\|\sqrt{r_{+} r_{-}} f\left(r_{+}^{2}, r_{-}^{2}\right)\right\|_{p}
$$

for $1 \leqq p \leqq 2, \frac{1}{p}+\frac{1}{p^{\prime}}=1$.

Since $v=\alpha v_{1}+(1-\alpha) v_{2}$, we write $\Gamma=\alpha \Gamma_{1}+(1-\alpha) \Gamma_{2}$, where $\Gamma_{i}=\Gamma_{i}(E, \eta)$ are defined in the same way as $\Gamma$ with $v$ replaced by $v_{i}, i=1,2$.

Thus

$$
\Gamma S \Gamma=\alpha^{2} \Gamma_{1} S \Gamma_{1}+\alpha(1-\alpha) \Gamma_{1} S \Gamma_{2}+\alpha(1-\alpha) \Gamma_{2} S \Gamma_{1}+(1-\alpha)^{2} \Gamma_{2} S \Gamma_{2},
$$

so

$$
\|\Gamma S \Gamma\| \leqq \alpha^{2}+2 \alpha(1-\alpha)+(1-\alpha)^{2}\left\|\Gamma_{2} S \Gamma_{2}\right\|=1-(1-\alpha)^{2}\left(1-\left\|\Gamma_{2} S \Gamma_{2}\right\|\right) .
$$


In view of (3.3), the proof of Lemma 3.1 in [6] gives

$$
\left\|\Gamma_{2} S \Gamma_{2}\right\| \leqq\left\|\Gamma_{2}\left(r_{+}^{2}, r_{-}^{2}\right)\right\|_{p}^{2} \leqq\left\|\hat{v}_{2}\left(r_{+}^{2}-r_{-}^{2}\right) e^{-\eta\left(r_{+}^{2}+r^{2}\right)}\right\|_{p}^{2}
$$

for any $2 \leqq p \leqq \infty$.

We now estimate

$$
\begin{aligned}
\left\|\hat{v}_{2}\left(r_{+}^{2}-r_{-}^{2}\right) e^{-\eta\left(r_{+}^{2}+r_{-}^{2}\right)}\right\|_{p}^{p} & =\int_{0}^{\infty} \int_{0}^{\infty}\left|\hat{v}_{2}\left(r_{+}^{2}-r_{-}^{2}\right)\right|^{p} e^{-\eta p\left(r_{+}^{2}+r_{-}^{2}\right)} d r_{+} d r_{-} \\
& =\frac{1}{4} \int_{0}^{\infty} \int_{0}^{\infty} \mid \hat{v}_{2}\left(r_{+}-r_{-}\right)^{p} e^{-\eta p\left(r_{+}+r_{-}\right)}\left(r_{+} r_{-}\right)^{-1 / 2} d r_{+} d r_{-} \\
& =\frac{1}{4} \int_{-\infty}^{\infty} d u \int_{|u|}^{\infty} d v\left|\hat{v}_{2}(u)\right|^{p} e^{-\eta p v}\left(v^{2}-u^{2}\right)^{-1 / 2},
\end{aligned}
$$

where we made the change of variables $u=r_{+}-r_{-}, v=r_{+}+r_{-}$.

If we now let $v=|u| \cosh \theta$, we get

$$
\frac{1}{4} \int_{-\infty}^{\infty} d u \int_{0}^{\infty} d \theta\left|\hat{v}_{2}(u)\right|^{p} e^{-\eta p|u| \cosh \theta} \leqq \frac{1}{4} \int_{-\infty}^{\infty} d u \int_{0}^{\infty} d \theta\left|\hat{v}_{2}(u)\right|^{p} e^{-(\eta / 2) p|u| e^{\theta}} .
$$

Making now $y=\eta p|u| e^{\theta}$, we have

$$
\begin{aligned}
& \left\|\hat{v}_{2}\left(r_{+}^{2}-r_{-}^{2}\right) e^{-\eta\left(r_{+}^{2}+r_{-}^{2}\right)}\right\|_{p}^{p} \\
& \quad \leqq \frac{1}{4} \int_{-\infty}^{\infty} d u \int_{\eta p|u|}^{\infty}\left|\hat{v}_{2}(u)\right|^{p} e^{-(y / 2)} y^{-1} d y \\
& \quad \leqq \frac{1}{4} \int_{-\infty}^{\infty} d u\left|\hat{v}_{2}(u)\right|^{p}\left[|\log \eta p| u|| e^{-(\eta / 2) p|u|}+\int_{0}^{\infty}|\log y| e^{-(y / 2)} d y\right] \\
& \quad \leqq \frac{1}{4} C|\log \eta| p^{-1 / \sigma}+D(p)
\end{aligned}
$$

for $p$ large enough by (1.1) and (1.2), where $\lim _{p \rightarrow \infty} D(p)=0$.

Let $p(\eta)=[C|\log \eta|]^{\sigma} \equiv C_{1}|\log \eta|^{\sigma}$. Then for $\eta$ small we have

$$
\left\|\hat{v}_{2}\left(r_{+}^{2}-r_{-}^{2}\right) e^{-\eta\left(r_{+}^{2}+r_{-}^{2}\right)}\right\|_{p(\eta)} \leqq(1 / 2)^{1 / p(\eta)}=e^{-C_{2}|\log \eta|^{-\sigma}}
$$

for some $C_{2}>0$.

Inequality (3.2) now follows from (3.4) and (3.5).

This finishes the proof of the Main Theorem if $\int v^{2} d v<\infty$. But since all our estimates are independent of this fact, it is not hard to see that this additional hypothesis can be removed by an approximation argument.

\section{Proof of Corollary 1}

In view of (1.4) it suffices to show that

$$
\mathbf{E}\left(|G(0, x ; E+i \eta)|^{2} \leqq C \eta^{-(2-\varepsilon)}\right.
$$

for some $\varepsilon>0$ and $C<\infty$ and $\eta$ sufficiently small. 
By the resolvent equation

$$
\begin{aligned}
G(0, x ; E+i \eta)= & G_{L}(0, x ; E+i \eta)-\frac{1}{2} G(0, L+1, E+i \eta) \\
& \times G_{L}(L, x ; E+i \eta)-\frac{1}{2} G(0,-L-1 ; E+i \eta) G_{L}(-L, x ; E+i \eta)
\end{aligned}
$$

for any $L$ such that $|x| \leqq L$.

Now, let $\beta>0, E_{0}>0$, and $|E| \leqq E_{0}$. We have

$$
\begin{aligned}
\mathbf{E}\left(\left|G_{L}(0, x ; E+i \eta)\right|^{2}\right)= & \mathbf{E}\left(\left|G_{L}(0, x ; E+i \eta)\right|^{2} ; \operatorname{dist}\left(E, \sigma\left(H_{L}\right)\right) \geqq e^{-L^{\beta}}\right) \\
& +\mathbf{E}\left(\mid G_{L}\left(0, x ; E+\left.i \eta\right|^{2} ; \operatorname{dist}\left(E, \sigma\left(H_{L}\right)\right)<e^{-L^{\beta}}\right)\right. \\
\leqq & e^{2 L^{\beta}}+\eta^{-2} e^{-\delta L^{\beta}}
\end{aligned}
$$

by (1.4) if $L$ is large enough.

Choosing $L$ such that $\eta^{((\delta / 2)+1)^{-1}}=e^{-L^{\beta}}$ we get

$$
\mathbf{E}\left(\left|G_{L}(0, x ; E+i \eta)\right|^{2}\right) \leqq 2 \eta^{-2((\delta / 2)+1)^{-1}} .
$$

Also, by (1.3),

$$
\begin{aligned}
& \mathbf{E}\left(|G(0, L+1 ; E+i \eta)|^{2}\left|G_{L}(L, x ; E+i \eta)\right|^{2}\right) \\
& \quad \leqq \eta^{-2} \mathbf{E}(\mid G(0, L+1, E+i \eta)) \leqq K \eta^{-4} \exp \left\{-m|\log \eta|^{-\sigma} L\right\} \\
& \quad=K \eta^{-4} \exp \left\{-m\left[2((\delta / 2)+1)^{-1}\right]^{1 / \beta}|\log \eta|^{1 / \beta-\sigma}\right\} \rightarrow 0,
\end{aligned}
$$

as $\eta \rightarrow 0$ if we pick $0<\beta$, such that $1 / \beta-\sigma>1$.

Since the third term can be similarly estimated, (4.1) follows from (4.2).

\section{Proof of Corollary 2}

By symmetry it suffices to estimate

$$
\mathbf{P}\left\{\left|G_{L}(0, L ; E)\right|>\exp \left(-d_{1} L^{(\sigma+1)^{-1}}\right)\right\}
$$

for some appropriate $d_{1}>0$.

Using Chebychev's inequality and (1.3), we get that

$$
\begin{aligned}
\mathbf{P} & \left\{\left|G_{L}\left(0, L ; E+i \exp \left(-c_{1} L^{(\sigma+1)^{-1}}\right)\right)\right|>\exp \left(-d_{1} L^{(\sigma+1)^{-1}}\right)\right\} \\
& \leqq \exp \left(2 d_{1} L^{(\sigma+1)^{-1}}\right) E\left(\left|G_{L}\left(0, L ; E+i \exp \left(-c_{1} L^{(\sigma+1)^{-1}}\right)\right)\right|^{2}\right) \\
& \leqq \exp \left\{\left(2 d_{1}+2 c_{1}-m c_{1}^{-\sigma}\right) L^{(\sigma+1)^{-1}}\right\}=\exp \left\{-c_{2} L^{(\sigma+1)^{-1}}\right\}
\end{aligned}
$$

with $c_{2}>0$ if $2\left(d_{1}+c_{1}\right) c_{1}^{\sigma}<m$.

By the first resolvent equation

$$
G_{L}(0, L ; E)=G_{L}(0, L ; E+i \eta)-i \eta\left[G_{L}(E) G_{L}(E+i \eta)\right](0, L) .
$$

Therefore,

$$
\begin{aligned}
& P\left\{\left|G_{L}(0, L, E)\right|>2 \exp \left(-d_{1} L^{(\sigma+1)^{-1}}\right)\right\} \\
& \leqq P\left\{\left|G_{L}(0, L, E+i \eta)\right|>\exp \left(-d_{1} L^{(\sigma+1)^{-1}}\right)\right\} \\
& \quad+P\left\{\left|\eta G_{L}(E) G_{L}(E+i \eta)\right|>\exp \left(-d_{1} L^{(\sigma+1)^{-1}}\right)\right\} .
\end{aligned}
$$


By taking $\eta=\exp \left(-c_{1} L^{(\sigma+1)^{-1}}\right)$ and $c_{1}>0, d_{1}>0$ as in (5.1) the first term in the right-hand side of (5.3) is bounded by $\exp \left(-c_{2} L^{(\sigma+1)^{-1}}\right)$. With this choice of $\eta$ the second term is estimated by:

$$
P\left\{\operatorname{dist}\left(E, \sigma\left(H_{L}\right)\right)<\exp \left(-\frac{1}{2}\left(c_{1}-d_{1}\right) L^{(\sigma+1)^{-1}}\right)\right\} \leqq \exp \left(-c_{3} L^{(\sigma+1)^{-1}}\right)
$$

with $c_{3}>0$ using (1.4) if $c_{1}-d_{1}>0$.

Since we can satisfy both conditions by taking $c_{1}=2 d_{1}$ small enough, this finishes the proof.

Acknowledgements. A.K. wants to express his gratitude for the hospitality of the Instituto de Física da Universidade de São Paulo, where part of this work was performed. F.M. wants to express his gratitude to the Department of Mathematics at U.C. Irvine for the warm hospitality and stimulating atmosphere.

\section{References}

1. Edwards, S.F.: A second quantization formulation for electrons in disordered systems. J.Phys. C 8, 1660 (1975)

Thouless, D.J.: Phase transitions for the one-dimensional Landau-Ginzburg system and their relation to the theory of polymers and of electrons in a random potential. J. Phys. C 8, 1803 (1975)

Nitzan, A., Freed, K.F., Cohen, D.H.: Renormalization group and critical localization. Phys. Rev. B 15, 4476 (1977)

Aharony, A., Imry, Y.: The mobility edge as a spin-glass problem. J. Phys. C 10, L 487 (1977); Schuster, H.: On a relation between the mobility edge problem and an isotropic $X Y$ model. Z. Phys. B 31, 99-104 (1978)

Wegner, F.: The mobility edge problem: Continuous symmetry and a conjecture. Z. Phys. B 35, 207 (1979)

Schafer, L., Wegner, F.: Disordered system with $n$ orbitals per site: Lagrange formulation, hyperbolic symmetry, and Goldstone modes. Z. Phys. B 38, 113 (1980)

Parisi, G.: Some remarks on the electronic states in disordered materials. J. Phys. A 14, 735 (1981)

McKane, A.J., Stone, M.: Ann. Phys. 131, 36-55 (1981)

Efetov, K.B., Larkin, A.I., Khmelnitskii, D.E.: Interaction of diffusion modes in the theory of localization. Sov. Phys. JETP 52, 568 (1980)

2. Parisi, G., Sourlas, N.: Self-avoiding walk and supersymmetry. J. Phys. Lett. 41, L403-L406 (1980)

3. McKane, A.J.: Reformulation of $n \rightarrow 0$ models using anticommuting scalar fields. Phys. Lett. 76 A, 22-24 (1980)

4. Efetov, K.B.: Supersymmetry method in localization theory. Sov. Phys. JETP 55, 514-521 (1982)

Efetov, K.B.: Supersymmetry and theory of disordered metals. Adv. Phys. 32, 53-127 (1983)

Parisi, G.: An introduction to the statistical mechanics of amorphous systems. In: Recent advances in field theory and statistical mechanics. Zuber, J., Stora, R. (eds.). Amsterdam: Elsevier 1984

5. Klein, A., Perez, J.F.: In preparation

6. Campanino, M., Klein, A.: A supersymmetric transfer matrix and differentiability of the density of states in the one-dimensional Anderson model. Commun. Math. Phys. 104, 227-241 (1986)

7. Fröhlich, J., Martinelli, F., Scoppola, E., Spencer, T.: Constructive proof of localization in the Anderson tight binding model. Commun. Math. Phys. 101, 21-46 (1985) 
8. Zygmund, A.: Trigonometric series. Vol. II, Cambridge: Cambridge University Press 1959. See Theorem XII.10.12

Brown, G., Hewitt, E.: Adv. Math. 37, 27-60 (1980)

9. Wegner, F.: Bounds on the density of states in disordered system. Z. Phys. B 44, 9-15 (1981)

10. Carmona, R., Klein, A., Martinelli, F.: Anderson localization for Bernoulli and other singular potentials. Commun. Math. Phys. (to appear)

11. Berezin, F.A.: The method of second quantization. New York: Academic Press 1966

12. Klein, A., Landau, L.J., Perez, J.F.: Supersymmetry and the Parisi-Sourlas dimensional reduction: A rigorous proof. Commun. Math. Phys. 94, 459-482 (1984)

13. Carmona, R.: Random Schrödinger Operators, to appear in Proc. 14th St. Flour Probability Summer School. Berlin, Heidelberg, New York: Springer

14. Spencer, T.: The Schrödinger equation with a random potential - a mathematical review, to appear in Proc. 1984 Les Houches Summer School

15. Kotani, S.: Private communication

Communicated by T. Spencer

Received January 17, 1986; in revised form May 20, 1986

Note added in proof. 1) In the Main Theorem, if $v=v_{2}$ (i.e., $\alpha=0$ ), the proof can be modified to give

$$
E\left(\left|G_{L}(0, x ; E+i \eta)\right|^{2}\right) \leqq K_{p}\|\hat{v}\|_{2 p}^{2} \eta^{-\left(2-p^{-1}\right)} \exp \left\{-m|\log \eta|^{-\sigma}|x|\right\}
$$

for any $1 \leqq p<\infty$ with $K_{p}<\infty$. In this case there is nothing to prove for Corollary 1 .

2) Lemma 3.2 is close to the best possible since we can show that the left-hand side of (3.2) converges to 1 as $\eta \downarrow 0$. 
